\title{
Bioefficacy of Animal Urine Based Formulations against Spodoptera litura (Fabricius) and Spilartia obliqua (Walker) at Pantnagar, Uttarakhand - A Novel Approach
}

\author{
Shilpi Naik and Ruchira Tiwari*
}

Department of Entomology, College of Agriculture, G. B. Pant University of Agriculture and Technology, Pantnagar-263145, U.S. Nagar, Uttarakhand, India

*Corresponding author

\section{Keywords}

Animal urine, Antifeedant, Cow urine, Growth parameters, Spodoptera, Spilarctia

Article Info

Accepted:

04 June 2018

Available Online:

10 July 2018

\section{A B S T R A C T}

To evaluate the bioefficacy of animal urine based formulations i.e. Cow urine collected from Desi, Holstein and Jersey breeds, Buffalo urine, Ox urine, Goat urine and Horse urine @ 20\% and animal urine based neem leaf extracts @5\% against Spodoptera litura and Spilarchia obliqua, laboratory experiments were conducted for the first time in Department of Entomology, GBPUAT, Pant Nagar, Uttarakhand during 2017. The growth and development studies clearly showed the efficacy of Desi cow urine, Goat Urine, Horse urine and all animal urine based neem leaf extracts against 7days old larvae of S. litura and $S$. obliqua as all larvae died in treated castor leaves. Among the other treatments, significantly less survival and reduce weights of larvae, pupae and adults were recorded with extended developmental period and very less growth index values in comparison to untreated control. Antifeedant activity of animal urine based formulations showed that Goat urine, Desi cow urine neem leaf extract(DCUNLE) and Goat urine neem leaf extract (GUNLE) were found with strong antifeedant action with preference index between (0.460.48 ) against $4^{\text {th }}$ instar larvae of $S$. litura whereas Goat urine, DCUNLE, GUNLE, Ox urine neem leaf extract (OUNLE), Baffalo urine neem leaf extract (BUNLE) and Horse urine neem leaf extract (HUNLE) showed strong antifeedant activity with preference indices $(0.26$ to 0.49$)$ against $4^{\text {th }}$ instar larvae of $S$. obliqua. Thus, these preliminary studies clearly demonstrated the potential of animal urine based formulations for ecofriendly management of noxious pests, S. litura and S. obliqua.

\section{Introduction}

The Tobacco caterpillar, Spodoptera litura (Fabricius) (Noctuidae: Lepidoptera) a key pest amongst the polyphagous pests distributed throughout tropical and subtropical parts of the world and causing damage to more than 150 species of host plants (Rao et al.,
1993 and Murugesan and Dhingra, 1995). It has been reported to feed on 112 cultivated crop plants belonging to 44 families out of which about 40 species are known from India (Mallikarjun et al., 2004). It has been reported to cause heavy yield loss throughout India (Ferry et al., 2004). The Bihar hairy caterpillar, Spilarctia obliqua (Walker) 
(Lepidoptera: Arctiidae), is a sporadic pest, widely distributed in India and can attack 126 plant species belonging to 25 families and thrives on about 25 weed species (Mandal and Bhattacharya, 2003).

The chemical pesticides have been used for several decades in controlling these noxious pests as they have a quick knock down effect. However, their indiscriminate use resulted in development of resistance in $S$. litura to insecticides, particularly pyrethroids and carbamates (Kranthi et al., 2002, Huang and Han, 2007 and Whalon et al., 2008). S. obliqua also showed certain levels of behavioral resistance to different class of insecticides (Mandal et al., 2013) hence successful control of these pests is to some extent is difficult, but traditional synthetic pesticides have been the most effective means of pest control (Pavela, 2012).

Several work has been conducted on different types of plant materials such as neem, jatropha, onion, ginger, turmeric, lantana etc. against $S$. litura and $S$. oblique under field and laboratory conditions (Patel and Gajjer, 2001, Purwar and Yadav, 2003, Getanjaly and Tiwari, 2014a,b). Indigenous products such as plant extracts and animal excreta such as cow urine, cow dung, bio gas etc. have emerged as an alternative to the chemicals pesticides in recent times. Several workers reported the use of cow urine and plant extracts for the control of insect pests of field crops (Purwar and Yadav, 2004, Gupta, 2005; Akash and Tiwari, 2010a,b; Tiwari et al., 2016). Therefore, considering the economic importance of these pests and to reduce the poisonous effects of pesticides in an agro-ecosystem, a novel study was carried out to evaluate the growth inhibitory and antifeedant effects of some animal urine based eco-friendly formulations against $S$ litura and $S$. obliqua under laboratory conditions.

\section{Materials and Methods}

The bioassay technique of leaf dip method was followed to find out the growth regulatory effects and antifeedant activity of different animal urine based formulations against $S$. litura and S. obliqua based on various growth parameters on treated castor leaves, The studies were conducted under laboratory conditions during 2017 in the Department of Entomology, GB Pant University of Agriculture and Technology, Pant Nagar, Uttarankhand.

\section{Culture of the test insects}

The cultures of $S$. litura and S. obliqua were raised in glass jars on leaves of castor, Ricinus communis Linn. The nucleus culture of the test insect larvae were collected from university premises and brought to the laboratory, were reared on fresh castor leaves till pupation and healthy pupae were procured for the next generation. The cultures were maintained at $27 \mathrm{C}$ and $70 \pm 5 \% \mathrm{RH}$. The adults obtained from above culture were released in separate glass jars $(21 \times 15 \mathrm{~cm})$ the walls of which were lined with white paper for egg laying. White paper strips were also kept in the jars for egg laying and muslin cloth and strips were checked daily for egg laying and eggs were removed and placed in a separate jar on fresh succulent castor leaves for hatching. The neonate larvae were reared on fresh castor leaves to maintain the test culture of both the tested insects.

\section{Collection of animal urine and preparation of plant extracts}

Different animal urine based formulations were prepared using Cow urine collected from Desi, Holstein and Jersey breeds, Buffalo urine, Ox urine, Goat urine and Horse urine @ $20 \%$ and animal urine based neem leaf extracts@5\% and evaluated against larval 
stages of S. litura and S. obliqua. The animal urine was collected from the dairy farm of the University while the cow urine based plant extracts were prepared by collecting the neem leaves from the University campus of Pant nagar. To prepare 5\% concentrations of animal urine based neem extracts, 50 grams of neem leaves were weighed by using a top balance, washed, dried under shade and macerated in the electrical grinder and dipped in $1000 \mathrm{ml}$ of water separately in the containers and were kept for fermentation for $24 \mathrm{hrs}$ and then filter by using muslin cloth.

\section{Bioassay to study growth and development of tested larvae}

The growth and development of 7days old larvae of $S$. litura and $S$ oblique on different treated leaf discs was evaluated by using nochoice test method as suggested by (Singh et al, 1995). Fresh castor leaf discs $\left(4 \times 4 \mathrm{~cm}^{2}\right)$ were treated with cow urine and plant extracts, separately, with the help of atomizer and placed in tilted orientation under ceiling fan air dried at room temperature in the laboratory to evaporate water from the leaf and kept in a petridishes lined with moist filter paper to maintain the humidity treatment wise separately. Thereafter, 10 larvae / replication of both insects were released separately, into each petri dish $(90 \mathrm{~mm}$ dia.) containing treated leaf disc and allowed to feed till pupation. Treated leaves were provided ad libitum till pupation. Each treatment was replicated thrice. In control, the leaf discs were dipped in distilled water and drip dried before being given to larvae. Observations were recorded on the different growth parameters such as larval weight, larval survival, larval and pupal period, pupal survival, per cent adult emergence and growth index using following formula:

Growth index $=$ Per cent adult emergence Total developmental period

\section{Bioassay for Feeding Inhibition (Antifeedant activity)}

The feeding inhibition and antifeedant activity of animal urine based formulations on freshly molted $4^{\text {th }}$ instar larvae was investigated through no- choice bioassay method using $4.0 \mathrm{~cm}^{2}$ leaf discs. The $4^{\text {th }}$ instar larvae were subjected to bioassay using leaf disc residue technique. All experiments were conducted at $29 \pm 0.5^{\circ} \mathrm{C}$ and $75 \pm 5 \% \mathrm{RH}$. The areas of leaf consumed by the larvae in 24 hours were recorded with the help of graph paper. The preference for the treated leaves by the tested larvae was examined by the leaf disc choice test (Jermy, 1961). For this purpose the leaf disc $\left(4 \mathrm{~cm}^{2}\right)$ of standard plant (castor) untreated or treated with different animal urine based formulations were used. The $6 \mathrm{~h}$ starved $4^{\text {th }}$ instar larvae were released in triplicated per treatment. The per cent feeding in each treatment over control was worked out using the following formula:

Per cent feeding $=$

Leaf area fed by the larvae

Initial leaf area given for feeding

$\%$ Feeding inhibition (FI) (Antifeedant activity) was calculated following Pande and Shrivastav (2003)

$\%$ Feeding inhibition $(\mathrm{FI})=\frac{\mathrm{C}-\mathrm{T}}{\mathrm{C}+\mathrm{T}} \quad \mathrm{X} 100$

Where $\quad \mathrm{C}=$ Consumption of control leaves $\mathrm{T}=$ Consumption of treated leaves

Preference index was calculated according to Kogan and Geoden, (1970)

Preference index $(\mathrm{C})=\frac{2 \mathrm{~A}}{\mathrm{M}+\overline{\mathrm{A}}}$

Where $\mathrm{C}=$ Preference index

$A=$ area eaten on the treated leaf

$\mathrm{M}=$ area eaten on the untreated leaf 
The antiffedant activity of each plant extracts will be worked out on the basis of preference indices (C-values) according to the following scale:

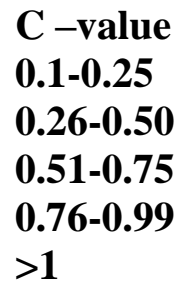

\section{Class Extremely antifeedant Strong antifeedant Moderately antifeedant Slightly antifeedant Preferred plant extract}

\section{Statistical analysis}

Data will be subjected to Complete Randomized Design (CRD) after suitable transformations. Mean data is analyzed by using square root transformation with adding factor 0.5 and per cent values were analyzed by using angular transformed values.

\section{Results and Discussion}

Effect of animal urine based formulations on growth and development of 7 days old larvae of $S$. litura

The data in Table 1 represented the efficacy of various animal urine formulations against 7 days old larvae of $S$. litura with the lowest larval survival (10.00\%) was observed in Goat urine followed by Horse urine $(13.33 \%)$ and Desi cow urine (16.67\%) with $90.00 \%$ larval survival in untreated control. A significant increase in larval period was observed in all treatments which were ranged from 9.54days in Jersey Cow urine to 11.73days in Ox urine in comparison to significantly less mean larval period (7.33 days) in untreated control. The mean larval weight was recorded the least in Desi cow urine $(148.40 \mathrm{mg}$ ) which was at par with Goat urine $(149.67 \mathrm{mg})$ followed by Horse urine $(154.75 \mathrm{mg}$ with the maximum larval weight $(600.88 \mathrm{mg})$ recorded in untreated control. No pupation was observed in Goat urine whereas very less pupation was observed in Horse urine $(10.00 \%)$ and Desi cow urine $(13.33 \%)$ with significantly maximum pupation in untreated control $(90.00 \%)$. The extended pupal period was observed among the treatments ranged from (9.50 days) in Jersey cow urine to11.00 days in Desi cow urine with 8.29days recorded in control. No adult emergence was observed in Desi cow urine, Goat urine and Horse uriner leaf discs. The mean pupal weight was ranged from $124.00 \mathrm{mg}$ in Horse urine to $170.38 \mathrm{mg}$ in Holstein cow urine with $301.37 \mathrm{mg}$ in untreated control. Only (6.67\%) adult emergence was observed in Ox urine followed by Holstein cow urine and Buffalo urine (20.00\%, each) and Jersey cow urine (26.67\%) in comparison to $90.00 \%$ adult emergence recorded in untreated. The mean adult weight was recorded the least in Ox urine (144.00mg) followed by Buffalo urine (153.50mg), Holstein cow urine (196.00mg) and Jersey cow urine $(200.12 \mathrm{mg})$ with the maximum adult weight $(218.70 \mathrm{mg}$ ) observed in untreated control. The growth index values couldn't be recorded for Desi cow urine, Goat urine and Horse urine treatments due to total larval mortality whereas very less growth index values were calculated for other animal urine treatments i.e. Ox urine (0.30), Buffalo urine (0.95), Holstein cow urine (1.00) and Jersey cow urine (1.40) with highest growth index value was calculated (5.76) in untreated control.

Effect of animal urine based neem leaf extractson growth and development of 7days old larvae of $S$. litura

The data based on the effect of neem leaf extracts prepared in various animal urine against 7 days old larva of $S$. litura is given in Table 2 which clearly represented the high efficacy of GUNLE with only(3.33\%) larval survival followed by HUNLE (6.67\%), DCULE and OUNLE (10.00\% each),with significantly highest larval survival $(90.00 \%)$ 
recorded in untreated control. A significant increase in larval period was observed in all animal based neem leaf extracts treatments i.e. ranged from 10.00days in DCUNLE to 11.00 days in GUNLE with the least larval period of 7.33 days were observed in untreated control. The larval weight was found significantly very less, ranged from (118.00mg) in GUNLE to (174.67mg) in HCUNLE with maximum larval weight $(600.88 \mathrm{mg})$ in untreated control. The all released 7 days old larvae were died so no pupation was observed in GUNLE followed by only $3.33 \%$ pupation occurred in DCUNLE and HUNLE with significantly highest pupation observed in untreated control $(90.00 \%)$. The pupal duration was found more in animal urine based neem leaf extracts treatments i.e., 10.00days in OUNLE to 11.50 days in DCUNLE with8.29 days in control. The mean pupal weight was again observed significantly very less ranged from $120.00 \mathrm{mg}$ in DCUNLE $(120.00 \mathrm{mg})$ to $182.67 \mathrm{mg}$ in JCUNLE with maximum pupal weight (301.37 mg) recorded in untreated control. All the pupae reared in castor leaves treated with DCUNLE, GUNLE, OUNLE and HUNLE were deformed and dead so no adult emergence was observed, The mean adult weight was calculated very less ranged from BUNLE(152.00mg) to JCUNLE (193.50mg) with maximum adult weight $(218.70 \mathrm{mg})$ in untreated control. The growth index values were found very less in BUNLE (0.15) followed by JCUNLE (0.48) and HCUNLE (0.62) with highest growth index value (5.76) in untreated control.

Effect of animal urine on growth and development of 7 days old larvae of $S$. obliqua

The data shown in Table 3 demonstrated that Ox urine gave the lowest larval survivability (16.67\%) followed by Goat urine $(20.00 \%)$, Desi cow urine (23.33\%), Horse urine (26.67\%)and Buffalo urine (30.00\%) with
93.33\% larval survival in untreated control. A significant increase in larval period was observed in all treatments ranged from 16.66days in Holstein Cow urine to 19.17.days in Goat urine with significantly less larval period (13.00 days) recorded in untreated control. In animal urine treated castor leaves, larval weight was observed very less which was ranged from $151.71 \mathrm{mg}$ in Desi cow urine to $209.81 \mathrm{mg}$ in Jersey cow urine with the maximum larval weight $(572.25 \mathrm{mg})$ recorded in untreated control. Similarly, a least pupation was recorded in Goat urine treated leaves $(3.33 \%)$ followed by Desi cow urine and Ox urine (13.33\% each), Buffalo urine $(16.67 \%)$. with maximum pupation $(93.33 \%)$ occurred in untreated control. The mean pupal period was found extended in all animal urine formulations which was ranged from 10.00days in Jersey cow urine to 11.38days in Goat urine treatments with the least pupal period (9.36days) recorded in untreated control. The mean pupal weight was also very less ranged from $101.00 \mathrm{mg}$ in Goat urine to $205.54 \mathrm{mg}$ in Jersey cow urine with maximum pupal weight $(299.85 \mathrm{mg})$ obtained in untreated control. No adult emergence was recorded in Goat urine treated leaves with the least adult emergence observed in Ox urine (3.33\%), Desi cow urine (6.67\%), Buffalo urine $(10.00 \%)$ and Horse urine $(16.67 \%)$ with comparatively higher adult emergence was recorded in untreated control $(90.00 \%)$. The adult weight was calculated very less in Desi cow urine $(155.00 \mathrm{mg})$ followed by Horse urine $(163.00 \mathrm{mg})$, Ox urine $(172.00 \mathrm{mg})$ and Buffalo urine $(183.00 \mathrm{mg})$ with whereas comparatively more mean adult weight (223.50mg) was recorded in untreated control. The growth index values were calculated very less for animal urine treatments which was ranged from Ox urine (0.12) to Jersey cow urine (1.38) in comparison to highest growth index value was calculated (4.03) in untreated control. 
Effect of animal urine based neem leaf extracts on growth and development of 7 days old larvae of $S$. obliqua

The data given in Table 4 clearly represented the high efficacy of GUNLE with only (6.67\%) larval survival followed by OUNLE $(10.00 \%)$ with significantly highest larval survival (93.33\%) recorded in untreated control. An extended larval period was again recorded from 17.25days in HCUNLE to 19.73days in GUNLE with minimum of 13.00days in untreated control. The significantly less mean larval weight was calculated in GUNLE (139.00mg) followed by OUNLE (150.00mg), DCUNLE (158.50mg), HUNLE (164.00) with maximum mean larval weight $(572.25 \mathrm{mg})$ in untreated control. A very less pupation ranged from (3.33\%) in GUNLE to $(23.33 \%)$ in HCUNLE was recorded with significantly highest pupation observed in untreated control (93.33\%). The mean pupal weight was recorded very less in GUNLE (73.00mg) followed by OUNLE (118.33mg), DCUNLE (151.50mg), BUNLE $(177.00 \mathrm{mg})$ with maximum pupal weight (299.85mg) observed in untreated control. A significantly very less adult weight ranged from $(149.00 \mathrm{mg})$ in DCUNLE to $(194.20 \mathrm{mg}$ ) in HCUNLE with maximum weight $(223.50 \mathrm{mg})$ of adult moths was calculated in untreated control. Similarly, the mean pupal weight was recorded the least in Goat urine (73.00mg) followed by Ox urine $(118.33 \mathrm{mg})$ and Desi cow urine $(151.50 \mathrm{mg})$ with maximum pupal weight $(299.85 \mathrm{mg})$ observed in untreated control. It has been clearly observed that the all pupae in DCUNLE, OUNLE and GUNLE were deformed and dead so no adult emergence was observed, while the adult emergence in other treatments was again significantly less which was ranged from (6.67\%) in HUNLE to $(20.00 \%)$ in JCUNLE with $90.00 \%$ in untreated control. A significantly very less adult weight ranged from $149.00 \mathrm{mg}$ in DCUNLE to $194.20 \mathrm{mg}$
HCUNLE was recorded with maximum weight adult $(223.50 \mathrm{mg})$ in untreated control. The growth index values were calculated again very less in HUNLE (0.22) followed by BUNLE (0.36), HCUNLE (0.61) and JCUNLE (0.68)with highest growth index value (4.03) in untreated control.

\section{Antifeedant activity of animal urine against} $4^{\text {th }}$ instar larvae of $S$. litura and $S$. obliqua

A glance at Table 5 showed that $S$. litura larvae fed very less $\left(4.77 \mathrm{~cm}^{2}\right)$ in Goat urine to $12.66 \mathrm{~cm}^{2}$ in Holstein cow urine treated castor leaf discs in comparison to untreated control where leaf discs were almost completely eaten $\left(15.26 \mathrm{~cm}^{2}\right)$. The significantly lowest mean per cent feeding was recorded in castor leaf discs treated with Goat urine $(29.81 \%)$ followed by Desi cow urine $(56.50 \%)$. The highest antifeedant activity $68.74 \%$ was recorded for Goat urine and $40.76 \%$ in Desi cow urine treated castor leaves. Similar trend was observed for feeding inhibition of tested larvae which was calculated the maximum (54.87\%)in Goat urine to $19.59 \%$ in Ox urine treated castor leaves. Overall mean preference index indicated that none of the animal urine were found to belong extremely strong antifeedant category but the preference indices calculated for Goat urine treated castor leaf discs were significantly less (0.48) which showed its strong antifeedant activity followed by Desi cow urine(0.74) and Buffalo urine (0.77) showed moderately antifeedant activity as compared to slightly antifeedant activity observed on other treated castor leaf discs treated with Ox urine, Horse urine, Jersey cow urine and Holstein cow urine $(0.80,0.83,0.85$ and 0.90 respectively).

The data depicted in Table 5 clearly showed that larvae of $S$. obliqua also fed very less in Goat urine $\left(5.18 \mathrm{~cm}^{2}\right)$ followed by Ox urine $\left(7.03 \mathrm{~cm}^{2}\right)$ and Desi cow urine $\left(8.36 \mathrm{~cm}^{2}\right)$ in comparison to control $\left(16.00 \mathrm{~cm}^{2}\right)$. The 
significantly lowest larval feeding on treated castor leaves was recorded in Goat urine $(32.38 \%)$ followed by Ox urine $(43.93 \%)$ and Desi cow urine $(52.25 \%)$ with $(100.00 \%)$ larval feeding was obtained in untreated control. Goat urine showed the highest antifeedant activity $(67.63 \%)$ followed by Ox urine $(56.06 \%)$, Desi cow urine $(47.77 \%)$ and Buffalo urine $(39.06 \%)$ whereas larval feeding inhibition was more in castor leaves treated with Goat urine $(51.09 \%)$ and Ox urine (38.94\%) followed by Desi cow urine $(31.36 \%)$ whereas In untreated control, leaf discs were almost completely eaten by the larvae. Overall mean preference index values clearly indicated that none of the animal urine formulations were found to belong extremely strong antifeedant category but the preference indices was significantly less in Goat urine (0.49) which showed its strong antifeedant activity whereas Ox urine (0.61)and Desi cow urine(0.68) showed moderately antifeedant activity as compared to slightly antifeedant activity observed on other treated castor leaf discs treated with Buffalo urine, Jersey cow urine,Horse urine and Holstein cow urine are $0.75,0.85,0.86$ and 0.96 respectively.

Antifeedant activity of animal urine based neem leaf extracts against $4^{\text {th }}$ instar larvae of $S$. litura

The data presented in Table 6 showed that the consumption of leaf discs by $S$. litura larvae was very less in DCUNLE $\left(4.63 \mathrm{~cm}^{2}\right)$ followed by GUNLE $\left(4.93 \mathrm{~cm}^{2}\right)$ and HUNLE $\left(6.83 \mathrm{~cm}^{2}\right)$ with maximum consumption in untreated control $\left(15.26 \mathrm{~cm}^{2}\right)$.

The significantly lowest mean larval feeding was recorded in DCUNLE (28.93\%) followed by GUNLE (30.81\%), HUNLE (42.68\%) with highest larval feeding in untreated control $(95.37 \%)$. The highest antifeedant activity $65.69 \%$ and $66.44 \%$ was recorded DCUNLE and GUNLE The maximum larval feeding inhibition (53.44\%) was calculated in DCUNLE followed by GUNLE (51.16\%), Overall mean preference index values indicated that none of the animal urine formulations were found to belong extremely antifeedant category but a strong antifeedant activity was noticed in DCUNLE (0.46) and GUNLE (0.48)whereas HUNLE(0.61) and BUNLE (0.63) and OUNLE (0.69) showed moderately antifeedant activity as compared to slightly antifeedant activity observed in JCUNLE (0.78)and HCUNLE (0.79)against $4^{\text {th }}$ instar larvae of $S$. litura.

The leaf areas consumed by the larvae of $S$. obliqua is given in Table 6 which showed that larvae fed very less leaf areas in Desi cow urine $\left(2.40 \mathrm{~cm}^{2}\right)$ followed by Horse urine $\left(2.83 \mathrm{~cm}^{2}\right)$ and Goat urine $\left(3.47 \mathrm{~cm}^{2}\right)$ treated castor leaves in comparison to control (16.00 $\mathrm{cm}^{2}$ ).

The significantly less mean larval feeding was observed in DCUNLE (15.00\%) and HUNLE $(17.68 \%)$ followed by GUNLE (21.68\%) with $100.00 \%$ larval feeding recorded in untreated control.

The highest antifeedant activity was calculated in DCUNLE (85.00\%) and HUNLE $82.31 \%$ ) followed by GUNLE (78.31\%).

The maximum per cent feeding inhibition of larvae was recorded in DCUNLE $(73.91 \%)$ followed by HUNLE (69.94\%), GUNLE $(64.36 \%)$. The overall mean preference index values indicated that none of the animal urine was belong to extremely antifeedant category but the strong antifeedant activity was noticed in DCUNLE (0.26), HUNLE(0.30), GUNLE(0.35), BUNLE (0.48), OUNLE (0.49), moderately antifeedant activity was shown by HCUNLE (0.72) whereas JCUNLE with preference index value $(0.81)$ showed the slight antifeedant activity against $4^{\text {th }}$ instar larvae of S.obliqua. 
Table.1 Effect of animal urine on growth and development of 7 days old larvae of Spodoptera litura

\begin{tabular}{|c|c|c|c|c|c|c|c|c|c|c|}
\hline Treatments & $\begin{array}{l}\text { Conc. } \\
(\%)\end{array}$ & $\begin{array}{l}\text { Larval } \\
\text { survival } \\
\%\end{array}$ & $\begin{array}{l}\text { Mean } \\
\text { larval } \\
\text { period } \\
\text { (days) }\end{array}$ & $\begin{array}{l}\text { Mean } \\
\text { larval } \\
\text { weight } \\
\text { (mg) }\end{array}$ & $\begin{array}{l}\text { Pupation } \\
(\%)\end{array}$ & $\begin{array}{l}\text { Mean } \\
\text { pupal } \\
\text { period } \\
\text { (days) }\end{array}$ & $\begin{array}{l}\text { Mean } \\
\text { pupal } \\
\text { weight } \\
\text { (mg) }\end{array}$ & $\begin{array}{l}\text { Adult } \\
\text { emergence } \\
(\%)\end{array}$ & $\begin{array}{l}\text { Mean } \\
\text { adult } \\
\text { weight } \\
\text { (mg) }\end{array}$ & $\begin{array}{l}\text { Growth } \\
\text { index } \\
\text { (G.I.) }\end{array}$ \\
\hline $\begin{array}{l}\text { Desi cow } \\
\text { urine }\end{array}$ & 20 & $\begin{array}{l}16.67 \\
(24.09)\end{array}$ & $\begin{array}{l}10.80 \\
(3.36)\end{array}$ & $\begin{array}{l}148.40 \\
(12.20)\end{array}$ & $\begin{array}{l}13.33 \\
(21.41)\end{array}$ & $\begin{array}{l}11.00 \\
(3.39)\end{array}$ & $\begin{array}{l}129.25 \\
(11.39)\end{array}$ & $\begin{array}{l}0.00 \\
(0.79)\end{array}$ & $\begin{array}{l}0.00 \\
(0.72)\end{array}$ & 0.00 \\
\hline $\begin{array}{l}\text { Holstein cow } \\
\text { urine }\end{array}$ & 20 & $\begin{array}{l}36.67 \\
(28.49)\end{array}$ & $\begin{array}{l}10.27 \\
(3.28)\end{array}$ & $\begin{array}{l}268.45 \\
(16.40)\end{array}$ & $\begin{array}{l}26.67 \\
(31.09)\end{array}$ & $\begin{array}{l}9.67 \\
(3.19)\end{array}$ & $\begin{array}{l}170.38 \\
(13.07)\end{array}$ & $\begin{array}{l}20.00 \\
(26.57)\end{array}$ & $\begin{array}{l}196.00 \\
(14.02)\end{array}$ & 1.00 \\
\hline $\begin{array}{l}\text { Jersey cow } \\
\text { urine }\end{array}$ & 20 & $\begin{array}{l}43.33 \\
(41.17)\end{array}$ & $\begin{array}{l}9.54 \\
(3.17)\end{array}$ & $\begin{array}{l}237.38 \\
(15.43)\end{array}$ & $\begin{array}{l}30.00 \\
(33.21)\end{array}$ & $\begin{array}{l}9.50 \\
(3.16)\end{array}$ & $\begin{array}{l}165.00 \\
(12.86)\end{array}$ & $\begin{array}{l}26.67 \\
(31.09)\end{array}$ & $\begin{array}{l}200.12 \\
(14.16)\end{array}$ & 1.40 \\
\hline Buffalo urine & 20 & $\begin{array}{l}30.00 \\
(33.21)\end{array}$ & $\begin{array}{l}10.80 \\
(3.36)\end{array}$ & $\begin{array}{l}179.00 \\
(13.40)\end{array}$ & $\begin{array}{l}23.33 \\
(28.88)\end{array}$ & $\begin{array}{l}10.20 \\
(3.28)\end{array}$ & $\begin{array}{l}0.00 \\
(0.73)\end{array}$ & $\begin{array}{l}20.00 \\
(26.57)\end{array}$ & $\begin{array}{l}153.50 \\
(12.40)\end{array}$ & 0.95 \\
\hline Ox urine & 20 & $\begin{array}{l}50.00 \\
(45.00)\end{array}$ & $\begin{array}{l}11.73 \\
(3.50)\end{array}$ & $\begin{array}{l}173.47 \\
(13.19)\end{array}$ & $\begin{array}{l}20.00 \\
(26.57)\end{array}$ & $\begin{array}{l}10.43 \\
(3.31)\end{array}$ & $\begin{array}{l}0.00 \\
(0.72)\end{array}$ & $\begin{array}{l}6.67 \\
(14.96)\end{array}$ & $\begin{array}{l}144.00 \\
(12.02)\end{array}$ & 0.30 \\
\hline Goat urine & 20 & $\begin{array}{l}10.00 \\
(18.43)\end{array}$ & $\begin{array}{l}11.00 \\
(3.39)\end{array}$ & $\begin{array}{l}149.67 \\
(12.25)\end{array}$ & $\begin{array}{l}0.00 \\
(0.60)\end{array}$ & $\begin{array}{l}0.00 \\
(0.72)\end{array}$ & $\begin{array}{l}0.00 \\
(0.73)\end{array}$ & $\begin{array}{l}0.00 \\
(0.65)\end{array}$ & $\begin{array}{l}0.00 \\
(0.74)\end{array}$ & 0.00 \\
\hline Horse urine & 20 & $\begin{array}{l}13.33 \\
(21.41)\end{array}$ & $\begin{array}{l}10.75 \\
(3.54)\end{array}$ & $\begin{array}{l}154.75 \\
(12.46)\end{array}$ & $\begin{array}{l}10.00 \\
(18.44)\end{array}$ & $\begin{array}{l}10.00 \\
(3.24)\end{array}$ & $\begin{array}{l}124.00 \\
(11.16)\end{array}$ & $\begin{array}{l}0.00 \\
(0.46)\end{array}$ & $\begin{array}{l}0.00 \\
(0.74)\end{array}$ & 0.00 \\
\hline $\begin{array}{l}\text { Untreated } \\
\text { Control }\end{array}$ & - & $\begin{array}{l}90.00 \\
(71.56)\end{array}$ & $\begin{array}{l}7.33 \\
(2.80)\end{array}$ & $\begin{array}{l}600.88 \\
(24.52)\end{array}$ & $\begin{array}{l}90.00 \\
(71.57)\end{array}$ & $\begin{array}{l}8.29 \\
(2.97)\end{array}$ & $\begin{array}{l}301.37 \\
(17.37)\end{array}$ & $\begin{array}{l}90.00 \\
(71.56)\end{array}$ & $\begin{array}{l}218.70 \\
(14.81)\end{array}$ & 5.76 \\
\hline SEM+ & & 1.550 & 0.002 & 0.004 & 0.107 & 0.002 & 0.005 & 0.154 & 0.003 & \\
\hline CD at $5 \%$ & & 4.648 & 0.005 & 0.013 & 0.324 & 0.006 & 0.014 & 0.460 & 0.010 & \\
\hline $\mathbf{C V}$ & & 7.581 & 0.081 & 0.128 & 0.645 & 0.123 & 0.092 & 1.232 & 0.067 & \\
\hline
\end{tabular}

Mean values are analyzed by square root transformation with 0.5 adding factor

Per cent values are angular transformed 
Table.2 Effect of animal urine based neem leaf extracts on growth and development of 7 days old larvae of Spodoptera litura

\begin{tabular}{|c|c|c|c|c|c|c|c|c|c|c|}
\hline Treatments & $\begin{array}{l}\text { Conc. } \\
(\%)\end{array}$ & $\begin{array}{l}\text { Larval } \\
\text { survival } \\
\%\end{array}$ & $\begin{array}{l}\text { Mean } \\
\text { larval } \\
\text { period } \\
\text { (days) }\end{array}$ & $\begin{array}{l}\text { Mean } \\
\text { larval } \\
\text { weight } \\
\text { (mg) }\end{array}$ & $\begin{array}{l}\text { Pupation } \\
(\%)\end{array}$ & $\begin{array}{l}\text { Mean } \\
\text { pupal } \\
\text { period } \\
\text { (days }\end{array}$ & $\begin{array}{l}\text { Mean } \\
\text { pupal } \\
\text { weight } \\
\text { (mg) }\end{array}$ & $\begin{array}{l}\text { Adult } \\
\text { emergence } \\
(\%)\end{array}$ & $\begin{array}{l}\text { Mean } \\
\text { adult } \\
\text { weight } \\
\text { (mg) }\end{array}$ & $\begin{array}{l}\text { Growth } \\
\text { index } \\
\text { (G.I.) }\end{array}$ \\
\hline $\begin{array}{l}\text { Desi Cow Urine Neem } \\
\text { Leaf Extract } \\
\text { (DCUNLE) }\end{array}$ & 5 & $\begin{array}{l}10.00 \\
(18.44)\end{array}$ & $\begin{array}{l}10.00 \\
(3.24)\end{array}$ & $\begin{array}{l}150.67 \\
(12.29)\end{array}$ & $\begin{array}{l}3.33 \\
(10.52)\end{array}$ & $\begin{array}{l}11.50 \\
(3.46)\end{array}$ & $\begin{array}{l}120.00 \\
(10.99)\end{array}$ & $\begin{array}{l}0.00 \\
(0.60)\end{array}$ & $\begin{array}{l}0.00 \\
(0.72)\end{array}$ & 0.00 \\
\hline $\begin{array}{l}\text { Holstein Cow Urine } \\
\text { Neem Leaf Extract } \\
\text { (HCUNLE) }\end{array}$ & 5 & $\begin{array}{l}20.00 \\
(26.57)\end{array}$ & $\begin{array}{l}10.83 \\
(3.37)\end{array}$ & $\begin{array}{l}174.67 \\
(13.24)\end{array}$ & $\begin{array}{l}20.00 \\
(26.57)\end{array}$ & $\begin{array}{l}10.75 \\
(3.35)\end{array}$ & $\begin{array}{l}167.17 \\
(12.95)\end{array}$ & $\begin{array}{l}13.33 \\
(21.41)\end{array}$ & $\begin{array}{l}192.75 \\
(13.90)\end{array}$ & 0.62 \\
\hline $\begin{array}{l}\text { Jersey Cow Urine } \\
\text { Neem Leaf Extract } \\
\text { (JCUNLE) }\end{array}$ & 5 & $\begin{array}{l}13.33 \\
(21.41)\end{array}$ & $\begin{array}{l}10.50 \\
(3.32)\end{array}$ & $\begin{array}{l}165.00 \\
(12.86)\end{array}$ & $\begin{array}{l}10.00 \\
(18.43)\end{array}$ & $\begin{array}{l}10.50 \\
(3.32)\end{array}$ & $\begin{array}{l}182.67 \\
(13.53)\end{array}$ & $\begin{array}{l}10.00 \\
(18.44)\end{array}$ & $\begin{array}{l}193.50 \\
(13.93)\end{array}$ & 0.48 \\
\hline $\begin{array}{l}\text { Buffalo Urine Neem } \\
\text { Leaf extract (BUNLE) }\end{array}$ & 5 & $\begin{array}{l}16.67 \\
(24.10)\end{array}$ & $\begin{array}{l}10.60 \\
(3.33)\end{array}$ & $\begin{array}{l}150.00 \\
(12.27)\end{array}$ & $\begin{array}{l}16.67 \\
(24.10)\end{array}$ & $\begin{array}{l}11.00 \\
(3.39)\end{array}$ & $\begin{array}{l}134.40 \\
(11.62)\end{array}$ & $\begin{array}{l}3.33 \\
(10.50)\end{array}$ & $\begin{array}{l}152.00 \\
(12.35)\end{array}$ & 0.15 \\
\hline $\begin{array}{l}\text { Ox Urine Neem Leaf } \\
\text { Extracts (OUNLE) }\end{array}$ & 5 & $\begin{array}{l}10.00 \\
(18.44)\end{array}$ & $\begin{array}{l}10.67 \\
(3.34)\end{array}$ & $\begin{array}{l}138.00 \\
(11.77)\end{array}$ & $\begin{array}{l}6.67 \\
(14.96)\end{array}$ & $\begin{array}{l}10.00 \\
(3.24)\end{array}$ & $\begin{array}{l}125.00 \\
(11.20)\end{array}$ & $\begin{array}{l}0.00 \\
(0.60)\end{array}$ & $\begin{array}{l}0.00 \\
(0.73)\end{array}$ & 0.00 \\
\hline $\begin{array}{l}\text { Goat Urine Neem Leaf } \\
\text { Extract (GUNLE) }\end{array}$ & 5 & $\begin{array}{l}3.33 \\
(10.51)\end{array}$ & $\begin{array}{l}11.00 \\
(3.39)\end{array}$ & $\begin{array}{l}118.00 \\
(10.90)\end{array}$ & $\begin{array}{l}0.00 \\
(0.52)\end{array}$ & $\begin{array}{l}0.00 \\
(0.72)\end{array}$ & $\begin{array}{l}0.00 \\
(0.72)\end{array}$ & $\begin{array}{l}0.00 \\
(0.79)\end{array}$ & $\begin{array}{l}0.00 \\
(0.73)\end{array}$ & 0.00 \\
\hline $\begin{array}{l}\text { Horse Urine Neem } \\
\text { Leaf extract (HUNLE) }\end{array}$ & 5 & $\begin{array}{l}6.67 \\
(14.97)\end{array}$ & $\begin{array}{l}10.75 \\
(3.35)\end{array}$ & $\begin{array}{l}140.00 \\
(11.85)\end{array}$ & $\begin{array}{l}3.33 \\
(10.51)\end{array}$ & $\begin{array}{l}11.00 \\
(3.39)\end{array}$ & $\begin{array}{l}122.00 \\
(11.07)\end{array}$ & $\begin{array}{l}0.00 \\
(0.52)\end{array}$ & $\begin{array}{l}0.00 \\
(0.73)\end{array}$ & 0.00 \\
\hline Untreated Control & - & $\begin{array}{l}90.00 \\
(71.56)\end{array}$ & $\begin{array}{l}7.33 \\
(2.80)\end{array}$ & $\begin{array}{l}600.88 \\
(24.52)\end{array}$ & $\begin{array}{l}90.00 \\
(71.53)\end{array}$ & $\begin{array}{l}8.29 \\
(2.96)\end{array}$ & $\begin{array}{l}301.37 \\
(17.37)\end{array}$ & $\begin{array}{l}90.00 \\
(71.57)\end{array}$ & $\begin{array}{l}218.70 \\
(14.80)\end{array}$ & 5.76 \\
\hline SEM+ & & 0.006 & 0.001 & 0.005 & 0.103 & 0.003 & 0.006 & 0.188 & 0.006 & \\
\hline CD at $5 \%$ & & 0.019 & 0.004 & 0.014 & 0.308 & 0.008 & 0.019 & 0.566 & 0.017 & \\
\hline $\mathbf{C V}$ & & 0.042 & 0.069 & 0.061 & 0.806 & 0.156 & 0.096 & 2.097 & 0.132 & \\
\hline
\end{tabular}

Mean values are analyzed by square root transformation with 0.5 adding factor

Per cent values are angular transformed 
Table.3 Effect of animal urine on growth and development of 7 days old larvae of Spilarctia oblique

\begin{tabular}{|c|c|c|c|c|c|c|c|c|c|c|}
\hline Treatments & $\begin{array}{l}\text { Conc. } \\
(\%)\end{array}$ & $\begin{array}{c}\text { Larval } \\
\text { survival } \\
(\%)\end{array}$ & $\begin{array}{l}\text { Larval } \\
\text { period } \\
\text { (days) }\end{array}$ & $\begin{array}{l}\text { Final mean larval } \\
\text { weight (mg) }\end{array}$ & $\begin{array}{l}\text { Pupation } \\
(\%)\end{array}$ & $\begin{array}{c}\text { Pupal } \\
\text { period } \\
\text { (days }\end{array}$ & $\begin{array}{l}\text { Mean pupal } \\
\text { weight (mg) }\end{array}$ & $\begin{array}{c}\text { Adult } \\
\text { emergence } \\
(\%)\end{array}$ & $\begin{array}{c}\text { Mean adult } \\
\text { weight } \\
\text { (mg) }\end{array}$ & $\begin{array}{c}\text { Growth } \\
\text { index } \\
\text { (G.I.) }\end{array}$ \\
\hline $\begin{array}{l}\text { Desi cow } \\
\text { urine }\end{array}$ & 20 & $\begin{array}{c}23.33 \\
(28.88)\end{array}$ & $\begin{array}{l}18.57 \\
(4.37)\end{array}$ & $\begin{array}{l}151.71 \\
(12.34)\end{array}$ & $\begin{array}{c}13.33 \\
(21.41)\end{array}$ & $\begin{array}{l}11.00 \\
(3.39)\end{array}$ & $\begin{array}{l}158.00 \\
(12.59)\end{array}$ & $\begin{array}{c}6.67 \\
(14.98)\end{array}$ & $\begin{array}{l}155.00 \\
(12.48)\end{array}$ & 0.23 \\
\hline $\begin{array}{l}\text { Holstein } \\
\text { cow urine }\end{array}$ & 20 & $\begin{array}{c}40.00 \\
(39.24)\end{array}$ & $\begin{array}{l}16.66 \\
(4.14) \\
\end{array}$ & $\begin{array}{l}198.67 \\
(14.11)\end{array}$ & $\begin{array}{c}30.00 \\
(33.21)\end{array}$ & $\begin{array}{l}10.25 \\
(3.42)\end{array}$ & $\begin{array}{l}194.33 \\
(14.46)\end{array}$ & $\begin{array}{c}30.00 \\
(33.20)\end{array}$ & $\begin{array}{l}208.78 \\
(14.47)\end{array}$ & 1.08 \\
\hline $\begin{array}{l}\text { Jersey cow } \\
\text { urine }\end{array}$ & 20 & $\begin{array}{c}53.33 \\
(46.91)\end{array}$ & $\begin{array}{l}17.68 \\
(4.26)\end{array}$ & $\begin{array}{l}209.81 \\
(14.50)\end{array}$ & $\begin{array}{c}43.33 \\
(41.16)\end{array}$ & $\begin{array}{l}10.00 \\
(3.45)\end{array}$ & $\begin{array}{l}205.54 \\
(14.40)\end{array}$ & $\begin{array}{c}40.00 \\
(39.24)\end{array}$ & $\begin{array}{l}206.83 \\
(14.40)\end{array}$ & 1.38 \\
\hline $\begin{array}{l}\text { Buffalo } \\
\text { urine }\end{array}$ & 20 & $\begin{array}{c}30.00 \\
(33.22)\end{array}$ & $\begin{array}{l}17.80 \\
(4.28)\end{array}$ & $\begin{array}{l}188.44 \\
(13.75)\end{array}$ & $\begin{array}{c}16.67 \\
(24.10)\end{array}$ & $\begin{array}{l}10.40 \\
(3.30)\end{array}$ & $\begin{array}{l}186.40 \\
(13.55)\end{array}$ & $\begin{array}{c}10.00 \\
(18.44)\end{array}$ & $\begin{array}{l}183.00 \\
(13.55)\end{array}$ & 0.35 \\
\hline Ox urine & 20 & $\begin{array}{c}16.67 \\
(24.09)\end{array}$ & $\begin{array}{l}18.11 \\
(4.31)\end{array}$ & $\begin{array}{l}174.20 \\
(13.22)\end{array}$ & $\begin{array}{c}13.33 \\
(21.42)\end{array}$ & $\begin{array}{l}11.22 \\
(3.28)\end{array}$ & $\begin{array}{l}179.25 \\
(13.13)\end{array}$ & $\begin{array}{c}3.33 \\
(10.51)\end{array}$ & $\begin{array}{l}172.00 \\
(13.15)\end{array}$ & 0.12 \\
\hline Goat urine & 20 & $\begin{array}{c}20.00 \\
(26.57)\end{array}$ & $\begin{array}{l}19.17 \\
(4.44)\end{array}$ & $\begin{array}{c}154.50 \\
(12.45)\end{array}$ & $\begin{array}{c}3.33 \\
(10.52)\end{array}$ & $\begin{array}{l}11.38 \\
(3.24) \\
\end{array}$ & $\begin{array}{c}101.00 \\
(0.73)\end{array}$ & $\begin{array}{c}0.00 \\
(0.90)\end{array}$ & $\begin{array}{c}0.00 \\
(0.73)\end{array}$ & 0.00 \\
\hline Horse urine & 20 & $\begin{array}{c}26.67 \\
(31.09)\end{array}$ & $\begin{array}{l}18.37 \\
(4.34)\end{array}$ & $\begin{array}{l}166.88 \\
(12.94)\end{array}$ & $\begin{array}{c}20.00 \\
(26.57)\end{array}$ & $\begin{array}{l}10.67 \\
(3.34) \\
\end{array}$ & $\begin{array}{c}160.33 \\
(4.75)\end{array}$ & $\begin{array}{c}16.67 \\
(24.10)\end{array}$ & $\begin{array}{c}163.00 \\
(12.79)\end{array}$ & 0.57 \\
\hline $\begin{array}{l}\text { Untreated } \\
\text { Control }\end{array}$ & - & $\begin{array}{l}93.33 \\
(7506)\end{array}$ & $\begin{array}{l}13.00 \\
(3.67)\end{array}$ & $\begin{array}{l}572.25 \\
(23.93)\end{array}$ & $\begin{array}{c}93.33 \\
(75.06)\end{array}$ & $\begin{array}{c}9.36 \\
(3.14)\end{array}$ & $\begin{array}{l}299.85 \\
(14.97)\end{array}$ & $\begin{array}{c}90.00 \\
(71.59)\end{array}$ & $\begin{array}{l}223.50 \\
(14.97)\end{array}$ & 4.03 \\
\hline SEM & & 0.007 & 0.001 & 0.006 & 0.008 & 0.001 & 1.42 & 0.062 & 0.007 & \\
\hline CD at $5 \%$ & & 0.021 & 0.004 & 0.019 & 0.025 & 0.004 & 4.26 & 0.185 & 0.021 & \\
\hline CV & & 0.032 & 0.060 & 0.035 & 0.046 & 0.068 & 22.23 & 0.401 & 0.099 & \\
\hline
\end{tabular}

DCUNLE-Desi Cow Urine Neem Leaf Extract JCUNLE- Jersey Cow Urine Neem Leaf Extract HCUNLEE-Holstein Cow Urine Neem Leaf Extract BUNLE-Buffalo Urine Neem Leaf extract OUNLE-Ox Urine Neem Leaf Extracts GUNLE-Goat Urine Neem Leaf Extract HUNLE- Horse Urine Neem Leaf extract; Mean values are analyzed by square root transformation with 0.5 adding factor

Per cent values are angular transformed 
Table.4 Effect of animal urine based neem leaf extracts on growth and development of 7 days old larvae of Spilarctia oblique

\begin{tabular}{|c|c|c|c|c|c|c|c|c|c|c|}
\hline Treatments & $\begin{array}{l}\text { Conc. } \\
(\%)\end{array}$ & $\begin{array}{l}\text { Larval } \\
\text { survival } \\
(\%)\end{array}$ & $\begin{array}{l}\text { Mean } \\
\text { larval } \\
\text { period } \\
\text { (days) }\end{array}$ & $\begin{array}{l}\text { Mean } \\
\text { larval } \\
\text { weight } \\
\text { (mg) }\end{array}$ & $\begin{array}{l}\text { Pupation } \\
(\%)\end{array}$ & $\begin{array}{l}\text { Pupal } \\
\text { period } \\
\text { (days) }\end{array}$ & $\begin{array}{l}\text { Mean } \\
\text { pupal } \\
\text { weight } \\
\text { (mg) }\end{array}$ & $\begin{array}{l}\text { Adult } \\
\text { emergence } \\
(\%)\end{array}$ & $\begin{array}{l}\text { Mean } \\
\text { adult } \\
\text { weight } \\
\text { (mg) }\end{array}$ & $\begin{array}{l}\text { Growth } \\
\text { index } \\
\text { (G.I.) }\end{array}$ \\
\hline $\begin{array}{l}\text { Desi Cow Urine Neem } \\
\text { Leaf Extract } \\
\text { (DCUNLE) }\end{array}$ & 5 & $\begin{array}{c}13.33 \\
(21.41)\end{array}$ & $\begin{array}{l}19.22 \\
(444)\end{array}$ & $\begin{array}{l}158.50 \\
(12.60)\end{array}$ & $\begin{array}{c}6.67 \\
(14.97)\end{array}$ & $\begin{array}{l}12.00 \\
(3.54)\end{array}$ & $\begin{array}{l}151.50 \\
(12.33)\end{array}$ & $\begin{array}{c}0.00 \\
(0.84)\end{array}$ & $\begin{array}{l}149.00 \\
(12.24)\end{array}$ & 0.00 \\
\hline $\begin{array}{l}\text { Holstein Cow Urine } \\
\text { Neem Leaf Extract } \\
\text { (HCUNLE) }\end{array}$ & 5 & $\begin{array}{c}30.00 \\
(33.21)\end{array}$ & $\begin{array}{l}17.25 \\
(4.21)\end{array}$ & $\begin{array}{l}202.67 \\
(14.25)\end{array}$ & $\begin{array}{c}23.33 \\
(28.88)\end{array}$ & $\begin{array}{l}10.22 \\
(3.27)\end{array}$ & $\begin{array}{l}197.86 \\
(14.07)\end{array}$ & $\begin{array}{c}16.67 \\
(24.10)\end{array}$ & $\begin{array}{l}194.20 \\
(13.95)\end{array}$ & 0.61 \\
\hline $\begin{array}{l}\text { Jersey Cow Urine } \\
\text { Neem Leaf Extract } \\
\text { (JCUNLE) }\end{array}$ & 5 & $\begin{array}{c}23.33 \\
(28.87)\end{array}$ & $\begin{array}{l}18.60 \\
(4.37)\end{array}$ & $\begin{array}{l}205.57 \\
(14.35)\end{array}$ & $\begin{array}{c}20.00 \\
(26.57)\end{array}$ & $\begin{array}{l}11.38 \\
(3.45)\end{array}$ & $\begin{array}{l}200.50 \\
(14.18)\end{array}$ & $\begin{array}{c}20.00 \\
(26.56)\end{array}$ & $\begin{array}{l}184.00 \\
(13.58)\end{array}$ & 0.68 \\
\hline $\begin{array}{l}\text { Buffalo Urine Neem } \\
\text { Leaf extract (BUNLE) }\end{array}$ & 5 & $\begin{array}{l}20.00 \\
(26.57)\end{array}$ & $\begin{array}{l}17.33 \\
(4.22)\end{array}$ & $\begin{array}{l}176.17 \\
(13.29)\end{array}$ & $\begin{array}{c}16.67 \\
(24.09)\end{array}$ & $\begin{array}{l}10.75 \\
(3.35)\end{array}$ & $\begin{array}{l}177.00 \\
(13.32)\end{array}$ & $\begin{array}{c}10.00 \\
(18.44)\end{array}$ & $\begin{array}{l}175.67 \\
(13.27)\end{array}$ & 0.36 \\
\hline $\begin{array}{l}\text { Ox Urine Neem Leaf } \\
\text { Extracts (OUNLE) }\end{array}$ & 5 & $\begin{array}{c}10.00 \\
(18.44)\end{array}$ & $\begin{array}{l}18.90 \\
(4.41)\end{array}$ & $\begin{array}{l}150.00 \\
(12.27)\end{array}$ & $\begin{array}{c}10.00 \\
(18.44)\end{array}$ & $\begin{array}{l}11.25 \\
(3.42)\end{array}$ & $\begin{array}{l}118.33 \\
(10.90)\end{array}$ & $\begin{array}{c}0.00 \\
(0.79)\end{array}$ & $\begin{array}{l}164.50 \\
(12.84)\end{array}$ & 0.00 \\
\hline $\begin{array}{l}\text { Goat Urine Neem Leaf } \\
\text { Extract (GUNLE) }\end{array}$ & 5 & $\begin{array}{c}6.67 \\
(14.97)\end{array}$ & $\begin{array}{l}19.73 \\
(4.50)\end{array}$ & $\begin{array}{l}139.00 \\
(11.81)\end{array}$ & $\begin{array}{c}3.33 \\
(10.51)\end{array}$ & $\begin{array}{l}12.70 \\
(3.64)\end{array}$ & $\begin{array}{l}73.00 \\
(8.57)\end{array}$ & $\begin{array}{c}0.00 \\
(0.98)\end{array}$ & $\begin{array}{c}0.00 \\
(0.72)\end{array}$ & 0.00 \\
\hline $\begin{array}{l}\text { Horse Urine Neem } \\
\text { Leaf extract (HUNLE) }\end{array}$ & 5 & $\begin{array}{c}16.67 \\
(24.09)\end{array}$ & $\begin{array}{l}18.50 \\
(4.36)\end{array}$ & $\begin{array}{l}164.00 \\
(12.86)\end{array}$ & $\begin{array}{c}13.33 \\
(21.41)\end{array}$ & $\begin{array}{l}11.67 \\
(3.49)\end{array}$ & $\begin{array}{l}155.75 \\
(12.50)\end{array}$ & $\begin{array}{c}6.67 \\
(14.96)\end{array}$ & $\begin{array}{l}172.25 \\
(13.14)\end{array}$ & 0.22 \\
\hline Untreated Control & - & $\begin{array}{c}93.33 \\
(75.03)\end{array}$ & $\begin{array}{l}13.00 \\
(3.68)\end{array}$ & $\begin{array}{l}572.25 \\
(23.93)\end{array}$ & $\begin{array}{c}93.33 \\
(75.03)\end{array}$ & $\begin{array}{c}9.36 \\
(3.14)\end{array}$ & $\begin{array}{l}299.85 \\
(17.33)\end{array}$ & $\begin{array}{c}90.00 \\
(71.57)\end{array}$ & $\begin{array}{l}223.50 \\
(14.97)\end{array}$ & 4.03 \\
\hline SEM+ & & 0.003 & 0.003 & 0.009 & 0.010 & 0.002 & 0.003 & 0.081 & 0.006 & \\
\hline CD at $5 \%$ & & 0.010 & 0.008 & 0.026 & 0.030 & 0.005 & 0.008 & 0.243 & 0.019 & \\
\hline CV & & 0.018 & 0.104 & 0.104 & 0.063 & 0.078 & 0.035 & 0.709 & 0.093 & \\
\hline
\end{tabular}

Mean values are analyzed by square root transformation with 0.5 adding factor

Per cent values are angular transformed 
Table.5 Comparative antifeedant activity of animal urine against $4^{\text {th }}$ instar larvae of Spodoptera litura and Spilartia obliqua (leaf area provided $=16 \mathrm{~cm}^{2}$ )

\begin{tabular}{|c|c|c|c|c|c|c|c|c|c|c|c|c|c|}
\hline \multirow[t]{2}{*}{ Treatments } & \multirow{2}{*}{$\begin{array}{l}\text { Co } \\
\text { nc. } \\
(\%)\end{array}$} & \multicolumn{6}{|c|}{ Spodoptera litura } & \multicolumn{6}{|c|}{ Spilartia obliqua } \\
\hline & & $\begin{array}{l}\text { Mean } \\
\text { leaf area } \\
\text { consume } \\
\mathrm{d}\left(\mathrm{cm}^{2}\right)\end{array}$ & $\begin{array}{l}\text { Feeding } \\
(\%)\end{array}$ & $\begin{array}{l}\text { Antifeedant } \\
\text { activity } \\
(\%)\end{array}$ & $\begin{array}{l}\text { Feeding } \\
\text { Inhibition } \\
(\%)\end{array}$ & $\begin{array}{l}\text { Preference } \\
\text { index* }\end{array}$ & $\begin{array}{l}\text { Antifeedant } \\
\text { Class }\end{array}$ & $\begin{array}{l}\text { MLA } \\
\mathrm{C} \\
\left(\mathrm{cm}^{2}\right)\end{array}$ & $\begin{array}{l}\text { Feedi } \\
\text { ng } \\
(\%)\end{array}$ & $\begin{array}{l}\text { Antifeeda } \\
\text { nt activity } \\
(\%)\end{array}$ & $\begin{array}{l}\text { Feeding } \\
\text { Inhibitio } \\
\mathrm{n}(\%)\end{array}$ & $\begin{array}{l}\text { Prefere } \\
\text { nce } \\
\text { index }\end{array}$ & $\begin{array}{l}\text { Antifeedant } \\
\text { Class }\end{array}$ \\
\hline $\begin{array}{l}\text { Desi cow } \\
\text { urine }\end{array}$ & 20 & $\begin{array}{l}9.04 \\
(3.09)\end{array}$ & 56.50 & 40.76 & 25.59 & 0.74 & $\begin{array}{l}\text { Moderately } \\
\text { antifeedant }\end{array}$ & $\begin{array}{l}8.36 \\
(2.98)\end{array}$ & 52.25 & 47.77 & 31.36 & 0.68 & $\begin{array}{l}\text { Moderately } \\
\text { antifeedant }\end{array}$ \\
\hline $\begin{array}{l}\text { Holstein } \\
\text { cow urine }\end{array}$ & 20 & $\begin{array}{l}12.66 \\
(3.63)\end{array}$ & 79.12 & 17.03 & 9.31 & 0.90 & $\begin{array}{l}\text { Slightly } \\
\text { antifeedant }\end{array}$ & $\begin{array}{l}14.90 \\
(3.93)\end{array}$ & 93.12 & 6.97 & 3.55 & 0.96 & $\begin{array}{l}\text { Slightly } \\
\text { antifeedant }\end{array}$ \\
\hline $\begin{array}{l}\text { Jersey cow } \\
\text { urine }\end{array}$ & 20 & $\begin{array}{l}11.30 \\
(3.44)\end{array}$ & 70.62 & 25.95 & 14.90 & 0.85 & $\begin{array}{l}\text { Slightly } \\
\text { antifeedant }\end{array}$ & $\begin{array}{l}11.94 \\
(3.53)\end{array}$ & 74.62 & 25.37 & 14.53 & 0.85 & $\begin{array}{l}\text { Slightly } \\
\text { antifeedant }\end{array}$ \\
\hline $\begin{array}{l}\text { Buffalo } \\
\text { urine }\end{array}$ & 20 & $\begin{array}{l}9.75 \\
(3.20)\end{array}$ & 60.93 & 36.10 & 22.03 & 0.77 & $\begin{array}{l}\text { Slightly } \\
\text { antifeedant }\end{array}$ & $\begin{array}{l}9.75 \\
(3.20)\end{array}$ & 60.93 & 39.06 & 24.27 & 0.75 & $\begin{array}{l}\text { Moderately } \\
\text { antifeedant }\end{array}$ \\
\hline Ox urine & 20 & $\begin{array}{l}10.26 \\
(3.28)\end{array}$ & 64.12 & 32.76 & 19.59 & 0.80 & $\begin{array}{l}\text { Slightly } \\
\text { antifeedant }\end{array}$ & $\begin{array}{l}7.03 \\
(2.75) \\
\end{array}$ & 43.93 & 56.06 & 38.94 & 0.61 & $\begin{array}{l}\text { Moderately } \\
\text { antifeedant }\end{array}$ \\
\hline Goat urine & 20 & $\begin{array}{l}4.77 \\
(2.50)\end{array}$ & 29.81 & 68.74 & 54.87 & 0.48 & $\begin{array}{l}\text { Strong } \\
\text { antifeedant }\end{array}$ & $\begin{array}{l}5.18 \\
(2.60) \\
\end{array}$ & 32.38 & 67.63 & 51.09 & 0.49 & $\begin{array}{l}\text { Strong } \\
\text { antifeedant }\end{array}$ \\
\hline Horse urine & 20 & $\begin{array}{l}10.88 \\
(3.37)\end{array}$ & 68.0 & 28.70 & 16.75 & 0.83 & $\begin{array}{l}\text { Slightly } \\
\text { antifeedant }\end{array}$ & $\begin{array}{l}12.08 \\
(3.54) \\
\end{array}$ & 75.50 & 24.50 & 13.96 & 0.86 & $\begin{array}{l}\text { Slightly } \\
\text { antifeedant }\end{array}$ \\
\hline $\begin{array}{l}\text { Untreated } \\
\text { Control }\end{array}$ & & $\begin{array}{l}15.26 \\
(3.97)\end{array}$ & 95.37 & - & - & - & & $\begin{array}{l}16.00 \\
(4.06)\end{array}$ & 100 & - & - & - & \\
\hline SEM+ & & 0.001 & & & & & & 0.002 & & & & & \\
\hline CD at $5 \%$ & & 0.002 & & & & & & 0.007 & & & & & \\
\hline CV & & 0.034 & & & & & & 0.120 & & & & & \\
\hline
\end{tabular}

Preference index values - 0.1-0.25 =Extremely antifeedant, $0.26-0.50=$ Strong antifeedant $\quad 0.51-0.75=$ Moderately antifeedant $0.76-0.99=$ Slightly antifeedant $>1=$ Preferred host 
Table.6 Comparative antifeedant activity of animal urine neem leaf extracts against $4^{\text {th }}$ instar larvae of Spodoptera litura and Spilartia obliqua (leaf area provided $=16 \mathrm{~cm}^{2}$ )

\begin{tabular}{|c|c|c|c|c|c|c|c|c|c|c|c|c|c|}
\hline \multirow{2}{*}{$\begin{array}{l}\text { Treatment } \\
\text { S }\end{array}$} & \multirow{2}{*}{$\begin{array}{l}\text { Con } \\
\text { c. } \\
(\%)\end{array}$} & \multicolumn{6}{|c|}{ Spodoptera litura } & \multicolumn{6}{|c|}{ Spilartia obliqua } \\
\hline & & $\begin{array}{l}\text { Mean } \\
\text { leaf area } \\
\text { consume } \\
\mathrm{d}\left(\mathrm{cm}^{2}\right)\end{array}$ & $\begin{array}{l}\text { Feedin } \\
\mathrm{g} \\
(\%)\end{array}$ & $\begin{array}{l}\text { Antifeeda } \\
\text { nt } \\
\text { activity } \\
(\%)\end{array}$ & $\begin{array}{l}\text { Feeding } \\
\text { Inhibitio } \\
\mathrm{n}(\%)\end{array}$ & $\begin{array}{l}\text { Preferen } \\
\text { ce index }\end{array}$ & $\begin{array}{l}\text { Antifeedant } \\
\text { Class }\end{array}$ & $\begin{array}{l}\text { MLAC } \\
(\mathrm{cm} 2)\end{array}$ & $\begin{array}{l}\text { Feeding } \\
(\%)\end{array}$ & $\begin{array}{l}\text { Antifeeda } \\
\text { nt activity } \\
(\%)\end{array}$ & $\begin{array}{l}\text { Feeding } \\
\text { Inhibition } \\
(\%)\end{array}$ & $\begin{array}{l}\text { Preference } \\
\text { index }\end{array}$ & $\begin{array}{l}\text { Antifeedant } \\
\text { Class }\end{array}$ \\
\hline $\begin{array}{l}\text { (DCUNLE } \\
\end{array}$ & 5 & $\begin{array}{l}4.63 \\
(2.27)\end{array}$ & 28.93 & 69.65 & 53.44 & 0.46 & $\begin{array}{l}\text { Strong } \\
\text { antifeedant }\end{array}$ & $\begin{array}{l}2.40 \\
(1.71)\end{array}$ & 15.00 & 85.00 & 73.91 & 0.26 & $\begin{array}{l}\text { Strong } \\
\text { antifeedant }\end{array}$ \\
\hline $\begin{array}{l}\text { (HCUNLE } \\
\text { ) }\end{array}$ & 5 & $\begin{array}{l}10.08 \\
(3.25)\end{array}$ & 63.00 & 33.94 & 20.44 & 0.79 & $\begin{array}{l}\text { Slightly } \\
\text { antifeedant }\end{array}$ & $\begin{array}{l}9.08 \\
(3.10)\end{array}$ & 56.75 & 43.25 & 27.59 & 0.72 & $\begin{array}{l}\text { Moderatelys } \\
\text { antifeedant }\end{array}$ \\
\hline (JCUNLE) & 5 & $\begin{array}{l}9.94 \\
(3.23)\end{array}$ & 62.12 & 34.86 & 21.11 & 0.78 & $\begin{array}{l}\text { Slightly } \\
\text { antifeedant }\end{array}$ & $\begin{array}{l}10.94 \\
(3.38)\end{array}$ & 68.37 & 31.62 & 18.78 & 0.81 & $\begin{array}{l}\text { Slightly } \\
\text { antifeedant }\end{array}$ \\
\hline (BUNLE) & 5 & $\begin{array}{l}7.11 \\
(2.75)\end{array}$ & 44.43 & 53.40 & 36.43 & 0.63 & $\begin{array}{l}\text { Moderately } \\
\text { antifeedant }\end{array}$ & $\begin{array}{l}5.11 \\
(2.37)\end{array}$ & 31.93 & 68.06 & 51.59 & 0.48 & $\begin{array}{l}\text { Strong } \\
\text { antifeedant }\end{array}$ \\
\hline (OUNLE) & 5 & $\begin{array}{l}8.16 \\
(2.94)\end{array}$ & 51.00 & 46.52 & 30.31 & 0.69 & $\begin{array}{l}\text { Moderately } \\
\text { antifeedant }\end{array}$ & $\begin{array}{l}5.16 \\
(2.38)\end{array}$ & 32.25 & 67.75 & 51.23 & 0.49 & $\begin{array}{l}\text { Strong } \\
\text { antifeedant }\end{array}$ \\
\hline (GUNLE) & 5 & $\begin{array}{l}4.93 \\
(2.44)\end{array}$ & 30.81 & 66.44 & 51.16 & 0.48 & $\begin{array}{l}\text { Strong } \\
\text { antifeedant }\end{array}$ & $\begin{array}{l}3.47 \\
(1.99)\end{array}$ & 21.68 & 78.31 & 64.36 & 0.35 & $\begin{array}{l}\text { Strong } \\
\text { antifeedant }\end{array}$ \\
\hline (HUNLE) & 5 & $\begin{array}{l}6.83 \\
(2.70)\end{array}$ & 42.68 & 55.24 & 38.16 & 0.61 & $\begin{array}{l}\text { Moderately } \\
\text { antifeedant }\end{array}$ & $\begin{array}{l}2.83 \\
(1.82)\end{array}$ & 17.68 & 82.31 & 69.94 & 0.30 & $\begin{array}{l}\text { Strong } \\
\text { antifeedant }\end{array}$ \\
\hline $\begin{array}{l}\text { Untreated } \\
\text { Control }\end{array}$ & & $\begin{array}{l}15.26 \\
(3.97)\end{array}$ & 95.37 & - & - & - & & $\begin{array}{l}16.00 \\
(4.06)\end{array}$ & 100 & - & - & - & \\
\hline SEM+ & & 0.001 & & & & & & 0.003 & & & & & \\
\hline CD at $5 \%$ & & 0.003 & & & & & & 0.010 & & & & & \\
\hline CV & & 0.061 & & & & & & 0.227 & & & & & \\
\hline
\end{tabular}

DCUNLE-Desi Cow Urine Neem Leaf Extract JCUNLE- Jersey Cow Urine Neem Leaf Extract HCUNLEE-Holstein Cow Urine Neem Leaf Extract

BUNLE-Buffalo Urine Neem Leaf extract OUNLE-Ox Urine Neem Leaf Extracts GUNLE-Goat Urine Neem Leaf Extract HUNLE- Horse Urine Neem Leaf extract

*Preference index values - 0.1-0.25 =Extremely antifeedant, $0.26-0.50=$ Strong antifeedant $\quad 0.51-0.75=$ Moderately antifeedant $0.76-0.99=$ Slightly antifeedant $>1=$ Preferred host 
Schoonhoven (1982), Sadek (2003) classified antifeedant substances into repellents (which repels an insect without making contact with the material), suppressants (which suppress biting activity after contact) or deterrents (which deter an insect from further feeding after ingestion of the plant material. Patel and Gajjar (2001) reported that neem leaves extract @5\% suspension gave 76.77 and 71.70 per cent larval mortality of $S$. litura. Purwar and Yadav, $(2003,2004)$ reported that the cow urine and decoction of neem leaves in cow urine were found promising to control $S$. litura and $S$. obliqua under field conditions at Pant nagar, India (Gupta, 2005). Neem in combination with cow urine was found effective in reducing the mustard aphid population with no adverse effect on coccinellid predators (Boomathi et al., (2006) conducted laboratory studies on the effect of neem seed kernel extract with cow urine and cow dung against Gram pod borer, Helicoverpa armigera and reported the toxicity of NSKE 5\%+cow urine 5\%+cow dung extract $5 \%$ against eggs and larvae of $\mathrm{H}$. armigera with low growth index values and high antioviposition and antifeedant index under laboratory conditions. Panwar and Chibber (2006) studied the effect of plant extracts on feeding potential of Spilarctia obliqua. They found leaf extracts of $A$. indica and Eucalyptus globule and green foliage of Lantana camara have suppressants and deterrents properties. However, strong repellant activity was noticed in case of neem only. Mala and Muthalagi (2008) reported that the larval duration of Spodoptera was increased when the fifth instar larvae were fed with $0.8 \%$ neem oil extract treated leaves. Sharma et al., (2009) reported that the aqueous extract of $A$. indica and $M$. azedarach resulted in statistically higher larvicidal (killing 20.9\% and 19.2\% larvae of $S$. litura. Pandey (2013) evaluated the antifeeding activity of leaf and seed extracts of the plant, Azadirachta indica against fourth instar larvae of $S$. litura and maximum antifeedant activity was observed in seed extract of A. indica at 5\% extract with antifeedant index of 100 per cent. Indiati (2014) reported that the neem seed, sugar apple leaf, and sugar apple seeds extract at a concentration of $50 \mathrm{~g} / \mathrm{l}(\mathrm{w} / \mathrm{v})$ were effective to suppress armyworm, $S$. litura populations (47-49\%) higher than chemical insecticides 2 cc/l (43\%). Akash Chand and Tiwari (2010) reported the effect of cow urine and different indigenous plant leaf extracts on food consumption and body weight of 10 days old $S$. Litura larvae and revealed that the larvae consumed significantly less leaf area when especially fed on neem extract treated leaves $(8.20 \mathrm{~cm} 2)$. Alexander et al, (2010) reported the efficacy of DDVP $(.025 \%)+$ NSKE+cow urine $2 \%$ against $S$. litura with higher larval mortality after $72 \mathrm{~h}$ of exposure. Geetanjaly and Tiwari (2014) conducted laboratory experiments to study the antifeedant and growth regulatory effects of cow urine, neem leaf extracts prepared in water and cow urine@ (5\% and $10 \%$ ) against different stages of Spilarctia obliqua. The cent per- cent mortality of neonate larvae of S. obliqua was observed with less mean leaf area consumed by 4th instarlarvae at higher concentration@ @ 10\%. Chaudhary et al., (2016) reported the efficacy of methanolic extracts of $C$. juncea, C. quinquefolia, curry leaf and garlic recorded above $80.00 \%$ feeding inhibition of S. oblique and larval mortality of $20.00 \%-44.44 \%$.

In conclusion, the experimental results of the present novel study clearly showed the superiority of all treatments to untreated control. Desi cow urine, Goat urine, Horse urine and animal urine based neem leaf extracts were showed growth regulatory and strong antifeedant activity against 7 days old and $4^{\text {th }}$ larvae of S. litura and S. obliqua. The present studies demonstrated that Desi cow urine, Ox urine, Goat urine, Buffalo urine and Horse urine alone and in combination with $5 \%$ neem leaf extracts can be used against insect pests as they are easily available, cost effective eco- friendly natural products. Therefore, it can be finally concluded that the animal urine based formulations has tremendous potential and possibilities to be included in overall pest management programme for the management of $S$. obliqua and $S$. litura but as being a preliminary and novel study made for the first 
time at Pantnagar, Uttarakhand, further in depth studies are essentially required to reach to the final conclusions.

\section{Acknowledgement}

We extend thanks to Director Experimentation Station, Dean Agriculture and Head, Department of Entomology, College of Agriculture, Govind Ballabh Pant University of Agriculture and Technology, Pantnagar for providing necessary facilities for these investigations.

\section{References}

Aakash Chand and Tiwari, R. (2010). Influence of cow urine and indigenous plant leaf extracts on feeding potential and larval weight of Spodoptera litura (Fabricius) (Noctuidae: Lepidoptera). Journal of Insect Science, 23(3): 313-317.

Aakash Chand and Tiwari, R. 2010. Effect of cow urine and some indigenous plant extracts on feeding preference of Spilarctia obliqua (Walker). Journal of Applied Entomology. 24(1):43-46

Alaxender, K., Verma, O.P. and Mishra. M.S. 2010. Evaluation of indigenous products with insecticide against tobacco caterpillar, Spodoptera litura infesting cabbage. Asian Journal of Bio Science, 4 (2): 199-201.

Boomathi, N., Sivasubramanian, P., and Raguraman, S. 2006. Biological activities of cow excreta with neem seed kernel extract against Helicoverpa armigera (Hubner). Annals of Plant Protection Sciences, 14(1): 11-16.

Chowdhury, H., Gotyal, B. S., Selvaraj, K. and Sarkar, S. K. 2016. Bioefficacy of plant extracts on stem rot, Macrophomina phaseolina (Tassi) Goid and Bihar hairy caterpillar, Spilosoma oblique (Walker) in jute crop. Journal of Applied and Natural Science, 8(1):191-195.

Ferry, N., Edwards, M.G., Gatehouse, J.A. and Gatehouse, A.M.R. 2004. Plant- insect interaction: molecular approaches to insect resistance (edited by Sasaki T, Christou P). Current Opinion in Biotechnology, 15:155-161.

Geetanjaly and Tiwari, R. 2014a. Antifeedant and growth regulatory effect of neem leaf extracts against Spilarctia obliqua (Walker). The Bioscan 9(3): 1151-1156 (Supplement on Plant Pathology). Geetanjaly and Tiwari, R. 2014b. Bioefficacy of cow urine based ecofriendly formulations against Spilarctia obliqua (Walker). Journal of Applied and Natural Science, 6(2): 680-686.

Gupta, M.P. 2005. Efficacy of neem in combination with cow urine against Mustard aphid and its effect on Coccinellid predators. Natural Product Radianc, 4(2): 102-106.

Huang, S.J. and Han, Z.J. 2007. Mechanisms for multiple resistances in field populations of common cutworm, Spodoptera litura (Fabricius) in China. Pesticide Biochemistry and Physiology, 87: 14-22.

Indiati, S. W. 2014. The use of sugar apple and neem extract to control leaf eating pest on soybean. Journal of Experimental Biology and Agricultural Sciences, 2(2): 208-214.

Kranthi, K.R., Jadhav, D.R., Kranthi, S., Wanjari, R.R., Ali, R.R. and Russell, D.A. 2002. Insecticide resistance in five major insect pests of cotton in India. Crop Protection, 21: 449- 460.

Mala, S. and Muthalagai, S. 2008. Effect of neem oil Extractive (NOE) on repellency, mortality, fecundity, development and biochemical analysis of Pericaliaricini (Lepidoptera: Arctidae). Journal of Biopesticide, 1: 63-66.

Mallikarjuna, N., Kranthi, K.R., Jadhav, D.R., Kranthi, S. and Chandra, S. 2004. Influence of foliar chemical compounds on the development of Spodoptera litura (Fabricius) in inter-specific derivatives of groundnut. Journal of Applied Entomology, 128: 321-328.

Mandal, P. and Bhattacharya, 
A.K.2003.Azaadirachtin, the most potent neem derivative: effect on Spilosoma obliqua. Indian Journal of Entomology, 65 (2):170-176.

Mandal. D, Bhowmik. P. and Baral K.2013. Evaluation of insecticides for the management of Bihar hairy caterpillar, Spilosoma obliqua(Walker) (Lepidoptera: Arctiidae) in black gram (Vigna mungo L.). Journal of Life Sciences, 8(2):429-431.

Murugesan, K. and Dhingara, S. 1995. Variability in resistance pattern of various groups of insecticides evaluated against Spodoptera litura (Fabricius) during a period spanning over three decades. Journal of Entomological Research, 19: 313-319.

Pande, Deepti and Srivastava, R.P. (2003). Toxicity and antifeedant activity of Indoxacarb (Avaunt 14.5 SC) against tobacco caterpillar, Spodoptera litura (Fab.).Insect Environment, 9: 69-70.

Panwar, S. S. and Chhibber, R. C. 2006. Influence of plant extract on feeding potential of Spilarctia obliqua. Indian Journal of Applied Entomology, 20(1):22-24

Patel, M.B. and Gajjar, S.N. 2001. Bioefficacy of various plant leaf extracts against Spodoptera litura (Fabricius) (Noctuidae: Lepidoptera). Indian Journal of Applied Entomology.15(2): 32-34.

Pavela, R. (2012). Efficacy of three newly developed botanical insecticides based on pongam oil against Plutella xylostella $(\mathrm{L})$ larvae. Journal of Biopesticides, 5(1), 62-70.
Purwar, J. P. and Yadav, S. R. 2003. Field efficacy of pest controlling agents from different origins against tobacco caterpillar, Spodoptera lituraon soybean. Indian Journal of Entomology, 65(3):382-385.

Purwar, J. P. and Yadav, S. R. 2004. Evaluation of age related response of Spilarctia obliqua to biorational insecticides. Annals of Plant Protection Sciences 12(2):271-274.

Rao, G.V.R., Wightman, J.A. and Rao, R.D.V. 1993. World review of the natural enemies and diseases of Spodopteralitura(Fabricius)

(Lepidoptera: Noctuidae). Insect Science and its Application, 14: 273284.

Sadek, M. M. 1997. Antifeedant and larvicidal effects of Eichhornia crassipes leaves on the cotton leafworm Spodoptera littoralis (Boisd.). Journal of Egyptian German Society of Zoology, 24: 209232.

Schoonhoven, L. M. 1982. Biological aspects of antifeedant to desert locust Schistocerca gregaria. Indian Journal of Entomology, 42(3):460-464.

Singh, C.P., Pandey, M.C., Pant, A.K., Lackchaura, B.D. (1995). Antifeedant properties of some indigenous plant extract against Helicoverpa armigera. Bioved., 6: 171-74.

Whalon, M.E., Mota-Sanchez, D. and Hollingworth, R.M. 2008. Global Pesticide Resistance in Arthropods, CABI, Cromwell Press, Trowbridge, UK, pp. 1-167.

\section{How to cite this article:}

Shilpi Naik and Ruchira Tiwari. 2018. Bioefficacy of Animal Urine Based Formulations against Spodoptera litura (Fabricius) and Spilartia obliqua (Walker) at Pantnagar, Uttarakhand - A Novel Approach. Int.J.Curr.Microbiol.App.Sci. 7(07): 387-402. doi: https://doi.org/10.20546/ijcmas.2018.707.048 